\title{
XCIV. The effect of the transition layer of a liquid on its surface tension
}

\author{
R.D. Kleeman D.Sc. B.A.
}

To cite this article: R.D. Kleeman D.Sc. B.A. (1912) XCIV. The effect of the transition layer of a liquid on its surface tension , Philosophical Magazine Series 6, 24:144, 876-885, DOI: 10.1080/14786441208634882

To link to this article: http://dx.doi.org/10.1080/14786441208634882

曲 Published online: 20 Apr 2009.

Submit your article to this journal $\lceil\pi$

Џ Article views: 6

Q View related articles $₫$ 
That this expression is practically true we can see from the following example.

We choose hydrogen as the ideal gas and get the normal volume $v_{1}=22.4452$ litres, which is a little too great. The volume of one gram-molecule of carbon dioxide at $0^{\circ}$ C. and 1 atmosphere is $v_{2}=22 \cdot 2635$ litres.

$$
\begin{gathered}
v_{0}{ }^{\prime} \text { for carbon dioxide is equal to } \frac{v_{2}}{v_{1}} . \\
\text { Consequently } \quad 1-v_{0}{ }^{\prime}=0.0081, \\
a=0.0134, \quad b=0.0054, \quad a-b=0.0080 .
\end{gathered}
$$

From the equation (9) it ought to be possible by trial to determine how $a$ and $b$ vary with temperature at constant pressure, at least when that pressure is not too high, because all the terms in the formula are measurable quantities.

From these lines it is furthermore seen that a series of the form (4) is identically the same as an equation of the form (1), $a$ and $b$ being any function of the temperature. It is evident that from a series in powers of $P$ it ought to be easy to determine by trial what kind of functions of the temperatare the coefficients are, thus getting the variation of $a$ and $b$ by aid of equation (4) for even higher pressures, at least inasmuch as the form of van der Waals' equation is true.

Furthermore, a series of the form (4) is more convenient to use by determining the volume than the solution of the cubic (1).

XCIV. The Effect of ithe Transition Layer of a Liquid on its Surface Tension. By R. D. KLEEMAN, D.Sc., B.A.*

TT requires the expenditure of energy to produce an 1 increase in the area of surface of a liquid. . The surfacetension is therefore the work done, keeping the temperature constant, in producing unit increase in the area of surface of the liquid. Now this increase of surface may be produced in $a$ variety of ways, since it follows from thermodynamics that the work done during an isothermal process between given limits is independent of the nature of the path of the process. One way suggested by Laplace is to suppose a slab of liquid cut into two parts by an imaginary plane, and the parts then separated by an infinite distance from one another. If $\mathrm{W}$ denote the total amount of work done during

* Communicated by the Author. 
the process of separation, and $A$ the amount of new surface formed, the surface-tension $\lambda_{2}$ is given by

$$
\lambda_{2}=\frac{\mathrm{W}}{\mathrm{A}} \cdot \cdot \cdot \cdot \cdot \cdot \cdot \cdot
$$

If the distribution of the molecules in the new surfaces formed is the same as in the interior of the liquid, and the law of molecular attraction were known, the work done could at once be calculated. But the distribution of the molecules in the surface of a liquid is not the same as in the interior, a transition layer is formed in which the density changes continuously from that of the liquid on one side of the layer to that of the vapour on the other side.

The nature of the effect of the formation of a transition layer on the surface tension can be easily investigated. Suppose that on separation of the two slabs of liquid in the foregoing process, no change takes place in the distribution of the molecules in the surfaces of the slabs. The work done, as before, is W. Now suppose the transition layers to be formed. This will require that the surface layer of the liquid undergoes expansion in different degrees in different parts. Since the complete process of increase of the surface of a liquid is a reversible one, we must suppose that the formation of the transition layer takes place in such a way that external work is done during the process, and that it is a reversible one. We may, for example, suppose that the liquid is contained in a cylinder in which the piston is in contact with the liquid surface in question exerting a pressure tending to prevent the formation of the layer. This work is done at the expense of the heat supplied since the temperature has to be kept constant during the process. Let $w$ denote the amount of work in this case. The actual surface-tension of a liquid is then given by

$$
\lambda_{1}=\frac{\mathrm{W}-w}{\mathrm{~A}} . \cdot . . . .
$$

Thus we see that the production of a transition layer on the surface of a liquid has the effect of decreasing the magnitude of the surface tension.

A formula will be developed in this paper which expresses $\mathrm{W}$ in terms of quantities which can be measured. Since $\lambda_{1}$ can also be measured, the value of $w$ can be calculated by means of equation (2).

Phil. Mag. S. 6. Vol. 24. No. 144. Dec. 1912. 3 M 
Let a mass of liquid represented by $A B$ in the figure be cut into two slabs by an imaginary plane $a b$. Let the

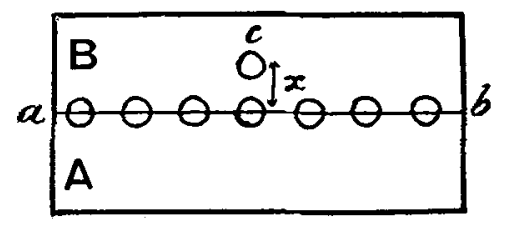

attraction of the slab $\mathrm{A}$ on a molecule $c$ at a distance $x$ from the plane $a b$ be denoted by $\mathrm{F}_{x}$. The amount of surface tension $\lambda_{x}$ contributed by the molecule on separation of the slabs, supposing no transition layer formed, is given by

$$
2 \lambda_{x}=\int_{\infty}^{x} \mathrm{~F}_{x} \cdot d x .
$$

Since the attraction between two molecules decreases with their distance of separation

$$
\left[\int \mathrm{F}_{x} \cdot d x\right]_{x=\infty}=0
$$

and hence

$$
2 \lambda_{x}=\int \mathrm{F}_{x} \cdot d x, \quad \text { or } 2 \frac{d \lambda x}{d x}=\mathrm{F}_{x} .
$$

The amount of intrinsic pressure $\mathbf{P}_{x}$ contributed by the molecule is equal to $F$, and hence the foregoing equation may also be written

$$
2 \frac{d \lambda_{x}}{d x}=\mathrm{P}_{x} \cdot \quad \cdot \quad \cdot \quad \cdot \quad \cdot \quad \cdot
$$

In order to be able to deal with all the molecules in the slab $\mathrm{B}$ in this manner, and to compare the results with one another, it is necessary to know the relative distribution of the mean positions of the molecules in a liquid. In a previous paper ${ }^{*}$ it was shown that we may suppose that the molecules in a pure liquid are situated at the points of intersection of three systems of parallel equidistant planes which intersect each other at right angles. Let us suppose that one of the planes coincides with the plane $a b$. Let us take one of the molecules in $a b$ as zero of coordinates. Consider the molecules lying on a line at right angles to $a b$

* Phil. Mag. April 1911, pp. 637-538. 
Transition Layer of a Liquid on its Surface Tension. $\quad 879$ and passing through the zero. We then have similarly as before

$$
\begin{gathered}
2\left\{\frac{d \lambda_{x}}{d x}+\frac{d \lambda_{2 x}}{d(2 x)}+\frac{d \lambda_{3 x}}{d(3 x)}+\ldots\right\}=2\left\{\frac{d \lambda_{x}}{d x}+\frac{1}{2} \frac{d \lambda_{2 x}}{d x}+\frac{1}{3} \frac{d \lambda_{3 x}}{d x}+\ldots\right\} \\
=\left(F_{x}+F_{2 x}+F_{8 x}+\ldots\right)
\end{gathered}
$$

where $x$ denotes the distance of separation of the molecules in the liquid. The intrinsic pressure $P_{n}$ of the liquid is given by

$$
P_{n}=\frac{1}{x^{2}}\left(\mathrm{~F}_{x}+\mathrm{F}_{2 x}+\mathrm{F}_{3 x}+\ldots\right), . . .
$$

and the surface-tension $\lambda_{2}$, on the supposition that no transition layer is formed, is given by

$$
\lambda_{2}=\frac{1}{x^{2}}\left(\lambda_{x}+\lambda_{2 x}+\lambda_{3 x}+\ldots\right), \text {. . . . . }
$$

where the factor $\frac{1}{x^{2}}$ denotes the number of rows of molecules standing on one $\mathrm{cm} .{ }^{2}$.

If the law of molecular attraction were exactly known we could express the quantities $\lambda_{x}, \lambda_{2 x}, \ldots$, in terms of the attraction constant of the molecules, that is, we could deduce a number of relations of the form

$$
\lambda_{n x}=\psi_{1}(n x, k), .
$$

where $k$ denotes the molecular attraction constant. On substituting for $\lambda_{n x}$ in equations (4) and (6) from the above equation, we obtain two equations from which $k$ may be eliminated. The resultant equation and equation (5) give an equation containing $\mathrm{P}_{n}, \lambda_{2}$, and $x$, only. The form of this equation depends on the form of the function $\psi_{1}(n x, k)$. A form of the equation which very approximately represents the facts is obtained from the following considerations.

Let us suppose that the attraction between two molecules is given by an expression of the form $\frac{k}{z^{m}}$, where $z$ denotes the distance of separation of the molecules, and $k$ and $m$ are constants. From equation (7) we then have

$$
\lambda_{\mu x}=\lambda_{x} c_{n} \text {. }
$$

where $c_{n}$ is a function of $n$ and $m$. Equations (4), (5), and $3 \mathrm{M} 2$ 
(6) then give

$$
\left\{\frac{1+\frac{c_{2}}{2}+\frac{c_{3}}{3}+\ldots}{1+c_{2}+c_{3}+\ldots}\right\} \frac{2}{x_{2}}\left\{\frac{d\left(\lambda_{2} x^{2}\right)}{d x}\right\}_{\mathrm{T}}=\frac{\mathrm{X} 2}{x^{2}}\left\{\frac{d\left(\lambda_{2} x^{2}\right)}{d x}\right\}_{\mathrm{T}}=\mathrm{P}_{n}
$$

But the attraction between two molecules is probably not given by an expression of the above form. $c_{n}$ is therefore probably a function of $x$ as well as of $n$ and $m$. But it will obviously not vary very much with $x$, since the law of attraction could always approximately be represented by an expression of the form given. The variation of the values of $c_{n}$ with $x$, it will be seen, affects the value of the function $\mathrm{X}$ very little on account of its form. It follows therefore that equation (9) very approximately represents the facts, and that the value of $\mathbf{X}$ can be determined with fair accuracy from a form of the law of molecular attraction which is approximately correct.

In previous papers * it was shown that the law of attraction between two molecules is approximately given by $\frac{k}{z^{5}}$, where $z$ denotes their distance of separation and $k$ a quantity which is constant at constant temperature. This law may therefore be used to calculate the value of $X$. The calculations will be facilitated by the following considerations. Let $a_{1}$ denote the work done against the attraction of the molecules in the plane $a b$ on moving the molecule situated in the slab $\mathrm{B}$ at a distance $x$ from the zero of coordinates to an infinite distance from the plane $a b$. Let $a_{2}$ denote the work done in moving the molecule situated at a distance $2 x$ from the zero of coordinates to an infinite distance from the plane, and $a_{3}$ the work done on transporting the molecule at a distance $3 x, \& c$. The total work $A_{1}$ done on moving the molecule at a distance $x$ to infinity against the attraction of all the molecules in the slab $A$ is therefore given by

$$
A_{1}=\left(a_{1}+a_{2}+a_{2}+\ldots a_{n}\right) \text {. }
$$

The total work $\mathrm{A}_{2}$ done on moving the molecule at a distance $2 x$ to infinity against the attraction of all the molecules in the slab $A$ is therefore given by

$$
\mathrm{A}_{2}=\left(a_{2}+a_{3}+a_{4}+\ldots a_{n}\right) ;
$$

* Phil. Mag. May 1910, pp. 795-807; Proc. Camb. Phil. Soc. vol. xvi. pt. 7 , pp. $586 \div 587$. 
Transition Layer of a Liquid on its Surface Iension. 881 and similarly

$$
\mathrm{A}_{3}=\left(a_{3}+a_{4}+a_{5}+\ldots a_{n}\right),
$$

and so on. We then have $2 \lambda_{n x}=A_{n}$, and from this equation, the special case $2 \lambda_{x}=A_{1}$, and equation (8), we obtain

$$
c_{n}=\frac{A_{n}}{A_{1}} \text {. . . . . . }
$$

It is necessary next to calculate the values of

$$
a_{1}, a_{2}, a_{3}, \ldots a_{n} \text {. }
$$

The component of attraction at right angles to the plane $a b$ of a molecule lying in the plane on a molecule in the slab $B$ at the distance $n x$ from the zero of coordinates is given by $\frac{k}{z^{5}} \frac{n x}{z}$, where $z$ denotes the distance of separation of the molecules. The coordinates of the latter molecule are $(n x, 0,0)$, and if those of the former be denoted by $(n x, w . x$, $v x)$ we have

$$
\varepsilon=\sqrt{\left(n^{2} x^{2}+w^{2} x^{2}+v^{2} x^{2}\right)},
$$

and the above expression becomes

$$
\frac{k n}{x^{5}\left\{n^{2}+w^{2}+v^{2}\right\}^{3}}
$$

The component of attraction $f_{n x}$ exerted by all the molecules in the plane $a b$ is therefore equal to

$$
\frac{k}{(n x)^{5}}+4 \sum_{w=1}^{w=\infty} \sum_{v=1}^{v=\infty} \frac{k n}{x^{5}\left\{n^{2}+w^{2}+v^{2}\right\}^{3}}+4 \sum_{v=1}^{v=\infty} \frac{k n}{x^{5}\left(n^{2}+v^{2}\right)^{3}} \text {. }
$$

The work $a_{n}$ required to move the molecule to infinity is given by

$\ddot{a_{n}}=\int f_{n x} d(n x)=x \frac{k}{4 n^{4} x^{4}}+\sum_{w=1}^{w=\infty} \sum_{v=1}^{v=\infty} \frac{k}{x^{4}\left\{n^{2}+w^{2}+v^{2}\right\}^{2}}+\sum_{v=1}^{v=\infty} \frac{k}{x^{4}\left(n^{2}+v^{2}\right)^{2}}$

On developing the right-hand side of this equation into a series (we need not retain the terms involving values of $v$ and $w$ higher than 3 ), the value of $a_{n}$ can readily be calculated for different values of $n$. The equation gives

$a_{1}=\frac{k}{x^{4}} \cdot 7532, \quad a_{2}=\frac{k}{x^{4}} \cdot 15195, \quad a_{3}=\frac{k}{x^{4}} \cdot 05056, \quad$ and $\quad a_{4}=\frac{k}{x^{4}} \cdot 0219$.

It is not necessary to obtain values of $a_{n}$ of a higher order than the fourth. 
We have accordingly

$\mathrm{A}_{1}=\cdot 9776 \frac{k}{x^{4}}, \mathrm{~A}_{2}=\cdot 2244 \frac{k}{x^{4}}, \mathrm{~A}_{3}=\cdot 07246 \frac{k}{x^{4}}$, and $\mathrm{A}_{4}=\cdot 0219 \frac{k}{x^{4}}$,

and hence equation (9) by means of equation (10) becomes

$$
\frac{\cdot 876 \times 2}{x^{2}}\left\{\frac{d\left(\lambda_{2} x^{2}\right)}{d x}\right\}_{\mathrm{T}}=\mathrm{P}_{n} \ldots . . .
$$

On comparing this equation-which represents the effect of all the molecules in the slab $B_{1}$ with equation (3) - which represents the effect of a single molecule, we see that the difference in form consists of the factor $\cdot 876$ occurring in one equation where the factor 1 occurs in the other. Since

$$
x=\left(\frac{m_{a}}{\rho}\right)^{1 / 3},
$$

where $\rho$ denotes the density of the substance and $m_{a}$ the absolute mass of a molecule, equation (11) may be written

$$
\frac{876 \times 6 \rho^{2}}{m_{a}^{1 / 3}}\left\{\frac{d}{d \rho}\left(\frac{\lambda_{2}}{\rho^{2 / 3}}\right)\right\}_{\mathrm{T}}=-\mathrm{P}_{n} . . .
$$

The foregoing equation may be thrown into a form which is more convenient. In a previous paper * it was shown that

$$
\mathrm{P}_{n}=\frac{d \mathrm{U}}{d v}=-\rho^{2} \frac{d \mathrm{U}}{d \rho},
$$

where U denotes the energy expended in overcoming the molecular attraction on separating the molecules of a gram of matter by an infinite distance from one another. The equation then becomes

$$
\frac{876 \times 6}{m_{a}^{1 / 3}} . d\left(\frac{\lambda_{2}}{\rho^{2 / 3}}\right)=d \mathrm{U} . . . \quad .
$$

Integrating it we obtain

$$
\frac{876 \times 6 \lambda}{m_{a}^{1 / 3} \rho^{2 / 3}}=\mathrm{U}+\mathrm{C}
$$

where $\mathrm{C}$ is an arbitrary constant. The vaiue of the constant $\mathrm{C}$ may be determined from the following considerations. When the density of the substance is infinitely small, or $x$ is infinitely great, $U=0$ and $\lambda_{2}=0$. The expression $\frac{\lambda}{\rho^{2 / 3}}$ may be written $\frac{K}{x^{8 / 3}}$, where $K$ is a constant, if we assume that the

* Proc. Camb. Phil. Soc. vol, xvi. pt. vi. pp. 543-546. 
Transition Layer of a Liquid on its Surface Tension. 883 attraction between two molecules is given by $\frac{k}{i^{5}}$. The expression therefore becomes equal to zero when the density of the substance becomes infinitely small. This holds whether the above expression for the molecular attraction is exactly true or not. Thus suppose that the molecular attraction is given by the expression $\frac{k}{z^{2}}$. The expression $\frac{\lambda_{2}}{\rho_{2 / 3}}$ then becomes $\frac{\mathrm{K}}{x^{1 / 3}}$, and thus as before becomes equal to zero when the density of the substance becomes infinitely small. But the molecular attraction which gives rise to surface-tension must decrease at a much greater rate with the distance of separation of the molecules than follows from the foregoing expression *, and $\frac{\lambda_{2}}{\rho^{2 / 3}}$ must therefore be equal to zero when the density of the substance is infinitely small. On applying the equation under consideration to matter of infinitely small density we obtain $\mathrm{C}=0$, and thus

$$
\cdot 876 \times 6 \lambda_{2}=\mathrm{U} m_{a}^{1 / 3} \rho^{2 / 3} .
$$

$U$ denotes the energy expended in overcoming the molecular attraction on separating the molecules of a gram of substance an infinite distance from one another, and if $u$ denotes the sum of the internal energies of the molecules, the internal heat of evaporation $\mathrm{L}$ is given by

$$
\mathrm{L}=\mathrm{U}_{1}-\mathrm{U}_{2}+u_{1}-u_{2},
$$

where the suffix 1 refers to the liquid and 2 to the saturated vapour. Let us assume that the internal energy of a molecule is independent of the vicinity of other molecules, in which case $u_{1}-u_{2}=0$. If the density of the saturated vapour is small in comparison with that of the liquid, as is the case at low temperatures, $\mathrm{U}_{2}=0$ and consequently $\mathrm{U}_{1}=\mathrm{L}$. Thus, on the above assumption, the values of $\lambda_{2}$ can be calculated for low temperatures of the liquid. This has been carried out for four liquids, the results being contained in the table given, which also contains the values of $\lambda_{1}$ obtained by Ramsay \& Shields $\dagger$. The values of $L$ were taken from a paper by Mills $\ddagger$. The values of $\lambda_{2}$ are greater than those of $\lambda_{1}$, as was indicated at the beginning of the paper. The difference $\left(\lambda_{2}-\lambda_{1}\right)$ is the external work done in the formation of the transition layer. It will be seen that it is

* Phil. Mag. Jan. 1911, pp. 89-90.

$\dagger$ Phil. Trans. of the Roy. Soc., A, vol. clxxxiv. p. 647 (1893).

$\ddagger$ Journ. of Phys. Chem. vol. viii. p. 405 (1904). 
884 Dr. R. D. Kleeman on the Effect of the

somewhat less than $\lambda_{1}$, and approximately independent of the temperature over a considerable range. The effect of the transition layer on the surface-tension is thus quite large.

\begin{tabular}{|c|c|c|c|c|c|c|c|c|c|c|c|}
\hline \multicolumn{6}{|c|}{ Ethyl oxide, $\mathrm{C}_{4} \mathrm{H}_{10} \mathrm{O}$. } & \multicolumn{6}{|c|}{ Carbon tetrachloride, $\mathrm{CCl}_{4}$. } \\
\hline $\mathrm{T}$ & $\mathbf{L}_{1}$. & $\rho$. & $\lambda_{2}$ & $\lambda_{2}$ & $\lambda_{2}-\lambda_{1}$. & T. & $\mathrm{L}_{1}$. & $\rho$. & $\lambda_{2}$ & $\lambda_{1}$. & $\lambda_{2}-\lambda_{1}$ \\
\hline $\begin{array}{l}313 \\
323 \\
333 \\
343 \\
353 \\
363 \\
373\end{array}$ & $\begin{array}{l}75 \cdot 36 \\
73 \cdot 01 \\
70 \cdot 79 \\
68 \cdot 35 \\
65 \cdot 85 \\
63 \cdot 31 \\
60 \cdot 33\end{array}$ & $\begin{array}{l}6894 \\
6764 \\
6658 \\
6532 \\
6402 \\
6250 \\
\cdot 6105\end{array}$ & $\begin{array}{l}23 \cdot 12 \\
22 \cdot 12 \\
21 \cdot 20 \\
20 \cdot 27 \\
19 \cdot 27 \\
18 \cdot 19 \\
17 \cdot 16\end{array}$ & $\begin{array}{r}14 \cdot 05 \\
12 \cdot 94 \\
11 \cdot 80 \\
10 \cdot 72 \\
9 \cdot 67 \\
8 \cdot 63 \\
7 \cdot 63\end{array}$ & $\begin{array}{l}9 \cdot 07 \\
9 \cdot 18 \\
9 \cdot 40 \\
9 \cdot 55 \\
9 \cdot 60 \\
9 \cdot 56 \\
9 \cdot 53\end{array}$ & $\begin{array}{l}363 \\
373 \\
383 \\
393 \\
403 \\
413 \\
423\end{array}$ & $\begin{array}{l}40 \cdot 62 \\
39 \cdot 68 \\
38 \cdot 64 \\
37 \cdot 63 \\
36 \cdot 58 \\
35 \cdot 56 \\
34 \cdot 42\end{array}$ & $\begin{array}{l}1 \cdot 4554 \\
1 \cdot 4343 \\
1 \cdot 4124 \\
1 \cdot 3902 \\
1 \cdot 3680 \\
1 \cdot 3450 \\
1 \cdot 3215\end{array}$ & $\begin{array}{l}26 \cdot 18 \\
\mathbf{2 5} \cdot 33 \\
24 \cdot 41 \\
\mathbf{2 3} \cdot 52 \\
22 \cdot 62 \\
21 \cdot 94 \\
20 \cdot 81\end{array}$ & $\begin{array}{l}17 \cdot 60 \\
16 \cdot 48 \\
15 \cdot 41 \\
14 \cdot 32 \\
13 \cdot 27 \\
12 \cdot 22 \\
11 \cdot 21\end{array}$ & $\begin{array}{l}8 \cdot 58 \\
8 \cdot 85 \\
9 \cdot 00 \\
9 \cdot 20 \\
9 \cdot 35 \\
9 \cdot 72 \\
9 \cdot 60\end{array}$ \\
\hline \multicolumn{6}{|c|}{ Methyl formate, $\mathrm{C}_{2} \mathrm{H}_{4} \mathrm{O}_{2}$. } & \multicolumn{6}{|c|}{ Benzene, $\mathrm{C}_{6} \mathrm{H}_{6}$. } \\
\hline $\begin{array}{l}303 \\
313 \\
323 \\
333 \\
343 \\
353 \\
363\end{array}$ & \begin{tabular}{|c|}
$107 \cdot 5$ \\
$103 \cdot 9$ \\
$99 \cdot 51$ \\
$95 \cdot 59$ \\
$92 \cdot 16$ \\
$88 \cdot 08$ \\
$85 \cdot 10$
\end{tabular} & $\begin{array}{l}.9598 \\
.9447 \\
.9294 \\
.9133 \\
.8968 \\
.8803 \\
.8636\end{array}$ & $\begin{array}{l}38 \cdot 35 \\
36 \cdot 64 \\
34 \cdot 74 \\
32 \cdot 98 \\
31 \cdot 94 \\
29 \cdot 65 \\
28 \cdot 25\end{array}$ & $\begin{array}{l}23 \cdot 09 \\
21 \cdot 56 \\
20 \cdot 05 \\
18 \cdot 58 \\
17 \cdot 55 \\
15 \cdot 70 \\
14 \cdot 29\end{array}$ & $\begin{array}{l}15 \cdot 26 \\
15.08 \\
14 \cdot 69 \\
14.40 \\
13.89 \\
13.95 \\
13.96\end{array}$ & $\begin{array}{l}353 \\
363 \\
373 \\
383 \\
393 \\
403 \\
413\end{array}$ & $\begin{array}{l}85 \cdot 62 \\
83 \cdot 74 \\
81 \cdot 98 \\
80 \cdot 05 \\
78 \cdot 12 \\
76 \cdot 10 \\
74 \cdot 09\end{array}$ & $\begin{array}{l}.8415 \\
.8041 \\
.7927 \\
.7809 \\
.7692 \\
.7568 \\
.7440\end{array}$ & $\begin{array}{l}29 \cdot 87 \\
28 \cdot 97 \\
28 \cdot 06 \\
27 \cdot 15 \\
26 \cdot 24 \\
25 \cdot 28 \\
24 \cdot 33\end{array}$ & $\begin{array}{l}20 \cdot 28 \\
19 \cdot 16 \\
18 \cdot 02 \\
16 \cdot 86 \\
15 \cdot 71 \\
14 \cdot 57 \\
13 \cdot 45\end{array}$ & $\begin{array}{r}9.59 \\
9.81 \\
10.04 \\
10.29 \\
10.53 \\
10.71 \\
10.88\end{array}$ \\
\hline
\end{tabular}

In previous papers a formula for the surface-tension $\lambda$ of a liquid was obtained on the supposition that no transition layer is formed and that the molecular attraction is given by the expression

$$
\phi\left(\frac{\mathrm{T}}{\mathrm{T}_{c}} \frac{x_{c}}{z}\right) \frac{\left(\Sigma \sqrt{m_{1}^{\prime}}\right)^{2}}{z^{5}}
$$

where $m_{1}^{\prime}$ denotes the atomic weight of an atom of a molecule. This gave

$$
\lambda=k\left(\frac{\rho}{m_{1}}\right)^{2}\left(\Sigma \sqrt{m_{1}^{\prime}}\right)^{2},
$$

where $m_{1}$ denotes the molecular weight relative to hydrogen, and $k$ is a quantity which has the same value for all substances at corresponding states. This equation was found to agree well with the facts. It follows, therefore, that each of the quantities $\lambda_{1}, \lambda_{2}$, and $\left(\lambda_{2}-\lambda_{1}\right)$ obeys the relation expressed by this equation.

The influence of the surrounding vapour of a liquid on its surface-tension may approximately be taken into account by supposing that we are dealing with a liquid of density $\left(\rho_{1}-\rho_{2}\right)$ which is not surrounded by vapour, where $\rho_{1}$ 
Transition Layer of a Liquid on its Surface Tension. 885 denotes the density of the liquid and $\rho_{2}$ that of the surrounding vapour. The surface-tension is thus approximately given * by

At low temperatures

$$
\lambda_{1}=32 \cdot 96\left(\rho_{\mathrm{t}}-\rho_{2}\right)^{4}\left(\frac{\sum \sqrt{m_{1}}{ }^{\prime}}{m_{1} \rho_{c}}\right)^{2} . . .
$$

$$
\mathrm{L}=\frac{2073 \rho_{1}^{2}}{m_{1}^{7 / 3} \rho_{c}^{2 / 3}}\left(\sum \sqrt{m_{1}}\right)^{2} \dagger
$$

and therefore more generally

$$
\mathrm{L}=\frac{2073\left(\rho_{1}-\rho_{2}\right)^{2}}{m_{1}^{7 / 3} \rho_{c}^{2 / 3}}\left(\Sigma v^{\prime} \overline{m_{1}{ }^{\prime}}\right)^{2}
$$

Equation (14) may therefore be written

$$
\lambda_{2}=203 \cdot 2\left(\rho_{1}-\rho_{2}\right)^{8 / 3} \frac{\left(\Sigma \sqrt{ } m_{1}\right)^{2}}{m_{1}^{2} \rho_{c}^{2 / 3}} . . . .
$$

Equation (14) may also be applied to mixtures. If two liquids 1 and 2 are mixed in the proportion of $n_{1}$ and $n_{2}$, and no new molecules are formed in the mixture, the value of $U$ is given by

$$
\mathrm{U}=\frac{\mathrm{L}_{1} n_{1}+\mathrm{L}_{2} n_{2}}{n_{1}+n_{2}}+\mathrm{H},
$$

where $H$ denotes the heat of solution per gram of solution, and $\mathrm{L}_{1}$ the internal heat of evaporation of a gram of liquid 1 , and $\mathrm{L}_{2}$ that of a gram of liquid 2 . The values of $\mathrm{L}_{1}, \mathrm{~L}_{2}$ and $H_{1}$ can usually be obtained and hence the value of $\lambda_{2}$ calculated.

The relative concentration of the ingredients in the transition layer of a mixture of substances is not the same as in the interior of the mixture. This property of a mixture is known as adsorption. Its thermodynamical treatment was first given by Willard Gibbs. It follows therefore that $\left(\lambda_{2}-\lambda_{1}\right)$ represents the external work done during the formation of the transition layer, and in altering the relative proportion of its ingredients. Equation (14) is therefore very useful, since it furnishes information on the nature of the transition layer in addition to that given by Gibbs's investigation. It may therefore help to clear up the important part played by the surfaces of contact in chemical changes.

London, July 10, 1912.

* Phil. Mag. Jan. 1911, pp. 99-101.

$\dagger$ Phil. Mag. Oct. 1910, pp. 686-68\%. 